\title{
Planejamento e Aplicação de Flipped Classroom para o Ensino de Teste de Software
}

\author{
Leo Natan Paschoal, ICMC - USP, paschoalln@usp.br \\ Simone R. S. Souza, ICMC - USP, srocio@icmc.usp.br
}

\begin{abstract}
Resumo: Em geral, os alunos de Computação não estão motivados para aprender teste de software. Eles preferem aprender programação em oposição a aprender a testar um programa. Diante disso, estudos mencionaram sobre investir em outros modelos pedagógicos, a fim de tornar o aprendizado mais atraente. O Flipped Classroom surge como uma proposta interessante, pois concentra-se no uso da sala de aula para a construção de conhecimento e não apenas para a transmissão de informações. No entanto, a adotação do modelo exige esforços por parte do professor, porque ele precisa preparar um conjunto de atividades a serem aplicadas eficientemente nos três momentos deste modelo. Além disso, o professor precisará estabalecer um conjunto de mecanismos de apoio a serem utilizados pelos alunos durante o ensino. A fim de contribuir para o ensino de teste de software, este artigo apresenta uma abordagem para apoiar o planejamento e a aplicação da Flipped Classroom para este domínio de ensino. Também são apresentados os resultados da aplicação da abordagem em uma aula de Engenharia de Software. Com base nos resultados, as lições aprendidas e as perspectivas futuras são apresentadas e discutidas.
\end{abstract}

Palavras-chave: Aprendizagem Ativa. Ensino de Computação. Teste de Software.

\section{Planning and Application of Flipped Classroom for Software Testing Teaching}

\begin{abstract}
In general, students of the Computer Science Programs are not motivated to learn software testing. They prefer learn programming in opposition to learn to test a program. Therefore, studies have mentioned investing in other pedagogical models in order to make learning more attractive. Flipped Classroom emerges as a good solution that focuses on the importance of the use of classroom for the construction of meaning rather than information transmission. However, the adoption of this model requires efforts by the professor, because the professor needs to prepare a set of activities to be efficiently applied during the three moments of this model. Also, the professor will need to establish a set of support mechanisms to be used by the students during the teaching. In order to contribute to the software testing teaching, this paper presents an approach to support the planning and the application of Flipped Classroom for this teaching domain. The paper presents the defined process and the results of its application in a Software Engineering Class. Based on results, lessons learned and future perspectives are presented and discussed.
\end{abstract}

Keywords: Active Learning. Computer Science Education. Software Testing.

\section{Introdução}

Teste de software é uma atividade do processo de desenvolvimento de software que tem como objetivo revelar os defeitos que estão presentes no software (MYERS; SANDLER; BADGETT, 2011). Apesar de sua relevância, estudos mencionam que os egressos dos cursos de Computação se formam com conhecimentos limitados sobre teste de software (LEMOS et al., 2018). Acredita-se que isso possa ter uma relação com a 
maneira com que os professores ensinam o conteúdo, uma vez que são poucas as iniciativas que utilizam metodologias de ensino diferentes da tradicional $^{1}$ (SOSKA; MOTTOK; WOLFF, 2016).

Apesar de existirem poucas iniciativas relatadas na literatura, existem alguns esforços para adoção de modelos pedagógicos que visam promover cenários que oportunizam a aprendizagem ativa aos alunos de teste de software (JR; ARCURI, 2018). Em experiências que objetivam promover aprendizagem ativa, os professores oferecem oportunidades para os alunos realizarem atividades mais práticas, semelhantes àquelas que eles enfrentarão no mercado de trabalho. Desse modo, os alunos precisam participar ativamente da disciplina, trabalhando em projetos mais próximos do "mundo real", solucionando problemas não triviais, trabalhando em equipe, dialogando com seus pares. Nesse tipo de abordagem, o professor deixa de ser um transmissor de conteúdos e passa a orientar os alunos, mediando e estimulando o aprendizado (HUNG, 2015).

Um modelo pedagógico que é fundamentado nas metodologias ativas de aprendizagem é o Flipped Classroom (BERGMANN; SAMS, 2012). Nesse modelo, o aluno estuda o conteúdo recomendado pelo professor a priori; em sala de aula ele soluciona as dúvidas com o professor e com os seus pares e, posteriormente, realiza atividades práticas com supervisão do professor (PASCHOAL; SILVA; SOUZA, 2017). O modelo visa mitigar alguns problemas enfrentados pelos alunos que aprendem com o método tradicional de ensino. No método tradicional, em sala aula os alunos assistem as preleções feitas pelo professor (atividade passiva) e em casa eles fazem trabalhos e realizam exercícios (atividade ativa). No entanto, as dúvidas sobre o conteúdo surgem principalmente quando os alunos estão realizando as atividades práticas, momento em que o professor não está disponível para saná-las (BERGMANN; SAMS, 2012). Diante disso, o modelo Flipped Classroom emerge, uma vez que preza pela realização de atividades ativas em sala de aula.

Apesar do modelo possuir uma proposta interessante, um dos principais desafios é implementá-lo (FASSBINDER et al., 2014). A implementação do modelo consiste em definir uma estratégia para utilizá-lo, preparar um conjunto de atividades e materiais didáticos para contemplar os três momentos que são previstos pelo modelo: antes da aula, durante a aula, depois da aula (BERGMANN; SAMS, 2012). Diante disso, o trabalho do professor se intensifica, fazendo com que ele gaste mais tempo para preparar uma aula (GANNOD; BURGE; HELMICK, 2008). Outra característica importante para adoção do modelo é definir quais conteúdos devem ser ensinados com apoio do modelo, uma vez que Flipped Classroom deve ser utilizado em algumas unidades da disciplina, não nela toda (FASSBINDER et al., 2014), porque nem todos os conteúdos são propícios de serem ensinados com Flipped Classroom (PASCHOAL; SILVA; SOUZA, 2017).

Uma área que merce investigação é o teste de software. No estudo de Paschoal e Souza (2018), os autores apresentam uma visão geral sobre as técnicas e critérios de teste de software que estão sendo ensinados por professores, em universidades no Brasil. A técnicas de teste funcioanl e seus critérios Particionamento em Classes de Equivalência e Análise do Valor Limite se destacam em relação aos demais assuntos. Acredita-se que o destaque está relacionado a simplicidade dos critérios e por isso é ensinada tanto em disciplina específica de Teste de Software quanto em disciplinas não específicas como Engenharia de Software. Embora a técnica seja abordada pelos professores, ela é ensinada por métodos tradicionais de ensino.

Este artigo visa contribuir com a implementação de Flipped Classroom no ensino de teste de software, apresentando uma abordagem para apoiar o planejamento e o

1 Entende-se por ensino tradicional, aquela abordagem em que o aluno não se prepara antes da aula e o professor ministra o assunto e, eventualmente, aplica uma atividade de fixação do conteúdo

(PASCHOAL; SILVA; SOUZA, 2017). 
uso de Flipped Classroom para esse domínio. A abordagem foi pré-definida levando em consideração os três momentos previstos pelo modelo. Ela é constituída por uma estratégia de implantação do modelo, um conjunto de mecanismos de apoio ao ensino do conteúdo e um conjunto de atividades. Após a definição da abordagem, ela foi instanciada e aplicada durante o ensino de teste de software em uma disciplina de Engenharia de Software. Após a experiência, os estudantes destacaram suas percepções a respeito da abordagem utilizada. Com base nessa experiência, alguns aprendizados e lições foram obtidas, contribuindo para o aprimoramento da abordagem proposta neste artigo.

Este artigo está estruturado da seguinte maneira. Na Seção 2 é apresentada uma visão geral do estado da arte sobre o planejamento de Flipped Classroom. Na Seção 3 é apresentado os detalhes a respeito do desenvolvimento da abordagem proposta. A Seção 4 apresenta a aplicação da abordagem e os resultados obtidos. Por fim, na Seção 5 são apresentados alguns aspectos que serão considerados para a evolução da abordagem e direcionamento para trabalhos futuros.

\section{Planejamento de Flipped Classroom: uma visão geral}

As pesquisas sobre Flipped Classroom destacam a importância do planejamento e documentação da estratégia que será utilizada para implementação do modelo.No estudo de Bergmann e Sams (2012), por exemplo, os autores discorrem sobre como planejar a aula, apresentando alguns direcionamentos para serem apreciados. Fassbinder et al. (2014) complementam esses direcionamentos a partir da exposição de algumas ferramentas que subsidiam a definição e a implementação do modelo. Gilboy, Heinerichs e Pazzaglia (2015), por fim, estruturaram um template para ser utilizado no planejamento da implementação do modelo, que oferece suporte aos três momentos previstos pelo modelo: (1) antes da aula, (2) durante a aula e (3) depois da aula).

De acordo com as recomendações de Bergmann e Sams (2012), o primeiro passo para adoção do modelo é a definição do objetivo da aula. Para estabelecer os diferentes objetivos de aprendizagem, o professor pode recorrer a Taxonomia de Bloom (TEIXEIRA, 2013). Se o professor optar por adotá-la, ele precisa elaborar um plano com o intuito de contemplar as seis categorias do domínio cognitivo (recordar, compreender, aplicar, analisar, avaliar, criar), nos três momentos previstos pelo modelo.

Após a definição dos objetivos, o professor precisa definir os mecanismos de apoio que serão utilizados para atender os objetivos que foram estabelecidos. Os mecanismos de apoio são utilizados pelos estudantes a priori a aula. O vídeo instrucional pode ser um mecanismo interessante de ser utilizado. $\mathrm{O}$ vídeo pode ser produzido pelo próprio professor que está planejando a estratégia de implementação do modelo ou por outros professores. Com o advento do YouTube e de outros repositórios de vídeos educacionais, a quantidade de vídeos com boa qualidade está em ascensão. Apesar do vídeo ser um dos mecanismos mais utilizados em implementações do modelo, outros mecanismos podem ser utilizados, pois a utilização de vídeo não caracteriza Flipped Classroom (BERGMANN; SAMS, 2012). O professor precisa analisar de forma cuidadosa se o vídeo é o mecanismo mais adequado

Para apoiar a definição dos mecanismos de apoio que serão recomendados aos alunos, o professor pode recorrer a teoria dos estilos de aprendizagem (FASSBINDER et al., 2014). Desse modo, o professor precisa utilizar algum instrumento para identificar os estilos de aprendizagem dos estudantes e determinar os mecanismos de apoio apropriados para os alunos da disciplina. Com a identificação dos estilos de aprendizagem, o professor pode planejar as atividades que desafiam os alunos a desenvolver suas capacidades (MOZZAQUATRO et al., 2009).

Quando o modelo é implantado em um conteúdo ou curso, o tempo disponível 
para realização de atividades em sala de aula é ampliado (GANNOD; BURGE; HELMICK, 2008). Diante disso, o professor precisa estar preparado para utilizar e explorar o tempo em sala de aula da melhor maneira possível (BERGMANN; SAMS, 2012). Ele pode estabelecer um conjunto de atividades para serem realizadas pelos estudantes em sala de aula. Esse conjunto de atividades deve oportunizar a compreensão com um nível maior de profundidade as complexidades dos conceitos. Nesse sentido, o professor pode conduzir atividades práticas, experimentos em laboratório, propor debates e discussões na turma, e até mesmo atividades que exploram a geração de conteúdo pelo próprio aluno.

$\mathrm{O}$ conjunto de mecanismos de apoio que serão recomendados pelo professor e o conjunto de atividades para a aula devem ser organizadas de acordo com os objetivos de aprendizagem. Diante disso, Gilboy, Heinerichs e Pazzaglia (2015) estabelecem um template para ajudar na organização da implementação do modelo. Os autores descrevem sugestões para uso do template, discorrendo que o professor deve certificar-se de que os objetivos de aprendizagem devem estar alinhados com as atividades e avaliações. $\mathrm{O}$ template é apresentado na Figura 1.

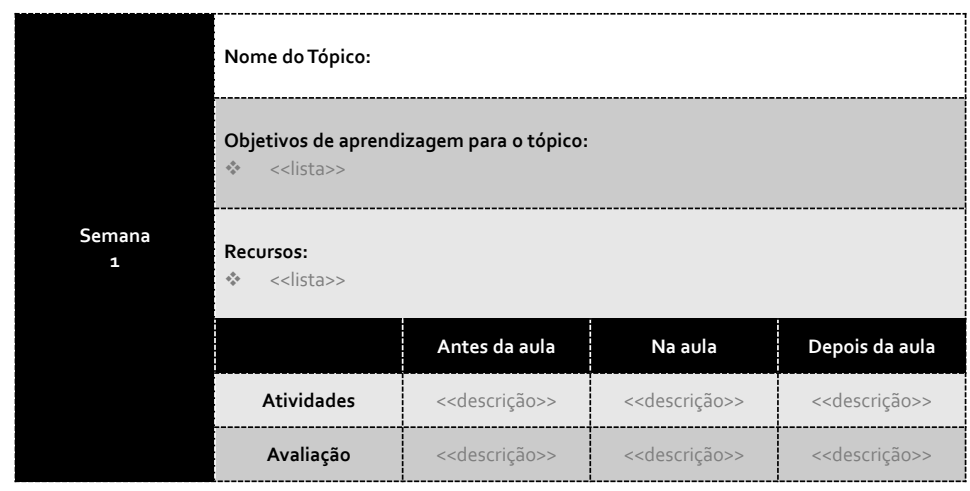

Figura 1 - Template para planejamento de Flipped Classroom. Adaptado de Gilboy, Heinerichs e Pazzaglia (2015).

Em relação ao template apresentado na Figura 1, no campo Recur sos, são estipulados os mecanismos de apoio que serão utilizados (e.g., slides, vídeos, jogos e demais recursos educacionais). No espaço Atividades, são prescritas as atividades que os alunos deverão realizar (e.g., trabalhos práticos, atividades de laboratório, produção de materiais, resolução de testes, dentre outras). Por fim, no campo Avaliação, são indicadas as atividades que serão utilizadas com o objetivo de avaliar o conhecimento do aluno.

\section{Flipped Classroom: uma contribuição à educação em Teste de Software}

Para a construção da abordagem, o primeiro passo foi a definição dos objetivos de aprendizagem para os conteúdos de teste de software ${ }^{2}$. Apesar de alguns autores indicarem a Taxonomia de Bloom para apoiar a definição dos objetivos de aprendizagem, foi estabelecido que os objetivos não seriam definidos utilizando a taxonomia de Bloom, pois nenhuma teoria, tendência ou princípio educacional seria adotado na abordagem. $\mathrm{Na}$ Figura 2 é possível observar os objetivos de aprendizagem para um tópico. Os objetivos educacionais para os demais tópicos, bem como o planejamento, estão disponíveis em: $<$ http://bit.ly/2Lkx2be>. Após a definição dos objetivos de aprendizagem, os mecanismos de apoio ao ensino de teste funcional foram selecionados. Para apoiar a definição dos

2 Dado que teste de software é uma área com uma ampla variedade de conteúdos, optou-se por escolher os conteúdos ensinados pelos professores no Brasil, a partir dos dados apresentados no estudo de Paschoal e Souza (2018). 
mecanismos e mitigar possíveis ameaças à validade da abordagem, foram selecionados mecanismos de apoio que passaram por um processo de avaliação.

Recorreu-se aos vídeos instrucionais disponibilizados em plataformas MOOCs (Massive Open Online Courses), uma vez que estudos mencionam que eles podem ser alternativas interessantes para aplicações do modelo Flipped Classroom, porque precisam possuir uma qualidade que atenda os critérios estabelecidos pela plataforma MOOC (FASSBINDER et al., 2014). Além disso, são vídeos com duração menor, em que um tópico é igual a um vídeo, em conformidade com o que é recomendado por Bergmann e Sams (2012). Diante disso, foram localizados cursos MOOC sobre teste de software que possuíam o conteúdo de teste funcional. Foram encontrados cursos nos idiomas português e inglês, oferecidos pelas plataformas Udemy ${ }^{3}$, Udacity ${ }^{4}$, Coursera ${ }^{5}$ e Edx ${ }^{6}$, criados por professores e/ou pesquisadores de renomadas instituições de ensino (SILVA; BARBOSA; FASSBINDER, 2017). Diante das opções encontradas, foram selecionados os vídeos do curso MOOC do Coursera em virtude dos mesmos possuírem instruções no idioma português (idioma nativo do público-alvo da abordagem). Os mecanismos estão listados na Figura 2, no campo Recursos.

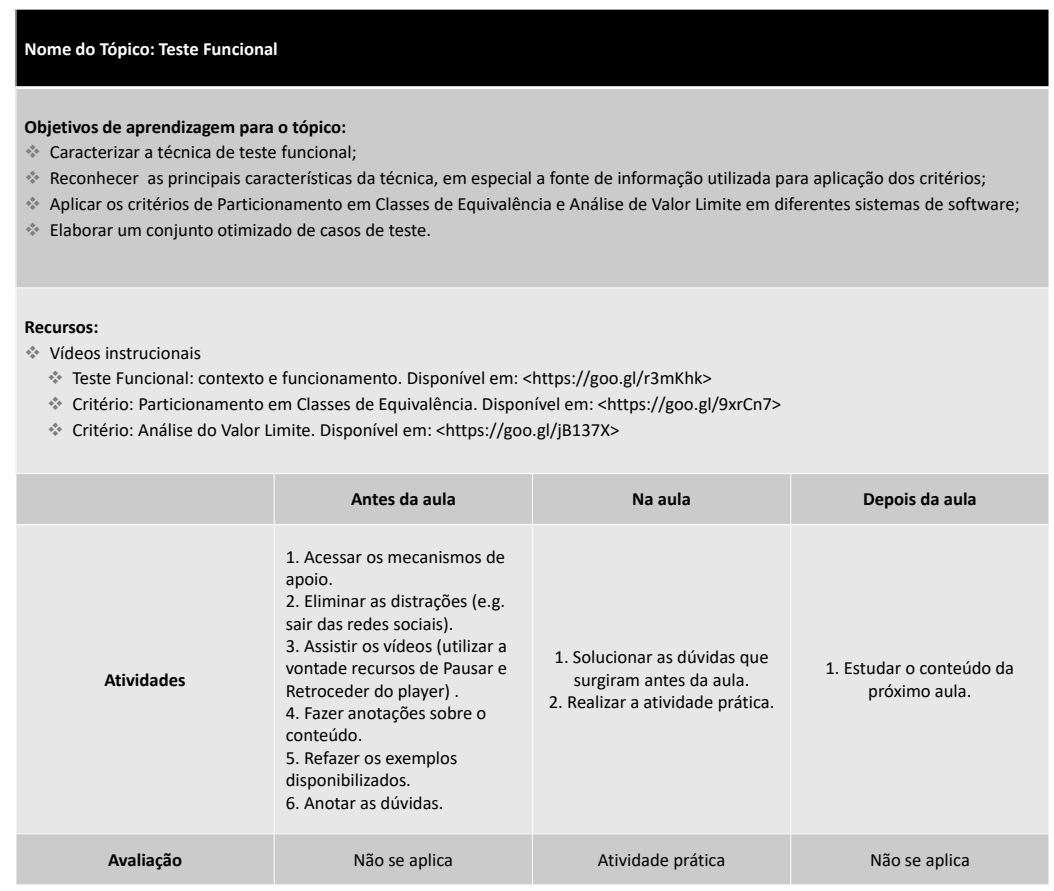

Figura 2 - Planejamento da implementação de Flipped Classroom para Teste Funcional

A partir da definição dos mecanismos de apoio, inicializou-se a etapa de definição de atividades que contemplam os três momentos previstos pelo modelo. Inicialmente, foram definidas as atividades que precisam ser disponibilizadas aos alunos no momento "antes da aula". Posteriormente, foram definidas as atividades para o momento "durante a aula". Durante a definição, foi selecionado um conjunto de atividades práticas que buscam atingir os objetivos de aprendizagem que foram estabelecidos para o tópico. Assim, na tentativa de selecionar atividades que exigem a aplicação prática dos conceitos presentes nos vídeos instrucionais, foram recorridos aos clássicos da literatura da área, em

\footnotetext{
http://bit.ly/2IEyUZq

http://bit.ly/2tGpCHW

http://bit.ly/2MA652N

6 http://bit.ly/21IB69j
} 
especial os trabalhos de Myers, Sandler e Badgett (2011), Delamaro, Jino e Maldonado (2016) e Maldonado, Delamaro e Vincenzi (2018).

Em relação ao momento "durante a aula", foi especificado como primeira atividade a resolução de dúvidas sobre o conteúdo estudado pelos alunos no momento "antes da aula". Também foi pré-estabelecido que a atividade prática seria utilizada como um critério para avaliar o conhecimento dos alunos. As atividades estão descritas na Figura 2, no campo Atividades, organizadas para cada momento previsto pelo modelo. Por fim, para o momento "depois da aula", foi definida a atividade que os alunos deveriam realizar nesse momento.

Para complementar a abordagem, após a definição do planejamento de implementação, foi estabelecida uma sequência de ações (Figura 3) que devem ser realizadas pelos sujeitos envolvidos no processo de ensino e aprendizagem de teste com o modelo Flipped Classroom (professor e aluno). Essa sequência é importante para deixar claro como usar o planejamento de implementação.

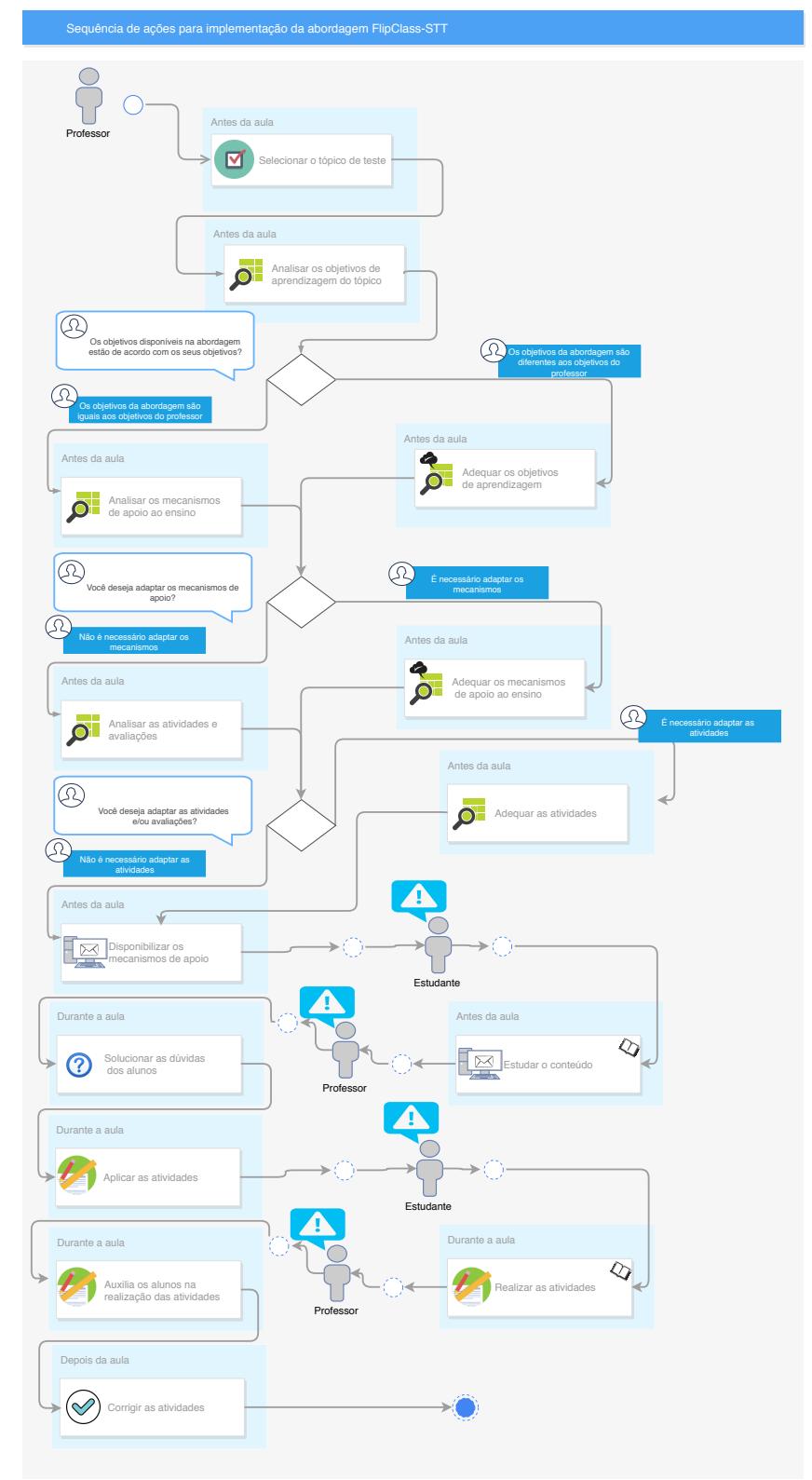

Figura 3 - Sequência de ações para implementação da abordagem. Versão maximizada disponível em: $<$ http://bit.ly/2QzeY3g> 


\section{Aplicação da abordagem}

Buscando analisar o impacto da abordagem no ensino de teste de software, a abordagem foi aplicada em uma aula da disciplina de Engenharia de Software, oferecida no $1^{\mathrm{O}}$ semestre de 2018, aos alunos do curso de Bacharelado em Ciências de Computação de uma Instituição de Ensino Superior. Trinta e seis alunos matriculados na disciplina participaram da experiência em estudar teste funcional com o modelo Flipped Classroom. A aplicação ocorreu no momento em que o conteúdo de teste de software estava sendo ministrado. Os conteúdos anteriores de Engenharia de Software (e.g., processos de desenvolvimento de software, engenharia de requisitos, modelagem, dentre outros) haviam sido ensinados por meio do método tradicional de ensino.

A sequência das ações para implementação da abordagem foi seguida, de modo que a abordagem original, sem modificações, fosse utilizada. Para apoiar a implementação e disponibilização dos mecanismos de apoio (vídeos instrucionais), foi utilizado o ambiente virtual de aprendizagem Moodle.

Tendo o objetivo de analisar a abordagem, um questionário ${ }^{7}$ foi preparado para ser aplicado ao final da utilização da abordagem. Esse questionário possui três perguntas e buscou coletar algumas informações a respeito da percepção dos alunos sobre a abordagem utilizada: (1) verificar se os mecanismos de apoio utilizados na abordagem contribuíram com o aprendizado antes da aula; (2) verificar se o tempo destinado à resolução de dúvidas no início da aula foi importante para o aluno; (3) indicar melhorias que precisam ser feitas na abordagem. O aluno precisava responder e justificar a resposta emitida.

Na primeira questão, 25 alunos $(69,33 \%)$ descreveram que os mecanismos de apoio (vídeos), utilizados antes da aula para estudo e compreensão dos conceitos teóricos sobre conteúdo, conseguiram contribuir com a aquisição de conhecimentos necessários para realização das atividades da abordagem. Ainda em relação a primeira questão, 11 alunos (31\%) mencionaram que os vídeos não foram suficientes para compreensão dos conceitos sobre o conteúdo e por isso não aprenderam suficientemente para a realização das atividades que fazem parte da abordagem. Alguns desses alunos justificaram o porquê de não terem adquirido os conhecimentos necessários. No entanto, apesar de concordarem, alguns alunos mencionaram problemas nos mecanismos que precisam ser melhorados. A Tabela 1 apresenta uma síntese das respostas.

Tabela 1 - Síntese das respostas emitidas pelos alunos na primeira questão

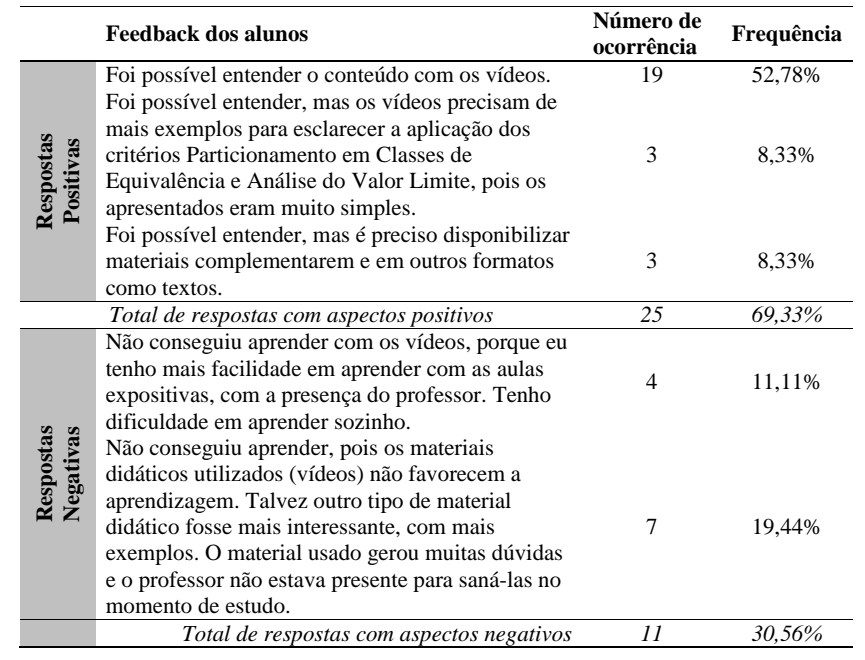

$7 \quad$ Disponível em: $<$ http://bit.ly/2LgyATL $>$ 
Na segunda pergunta, os alunos foram questionados sobre a importância dos minutos de discussão para resolução de dúvidas. Trinta e três alunos $(91,67 \%)$ descreveram que a discussão foi extremamente importante, porque dúvidas surgiram durante o estudo do conteúdo (antes da aula). Esses alunos também apresentaram alguns argumentos a favor do momento destinado a resolução de dúvidas. Além disso, 3 alunos $(8,33 \%)$ descreveram que esse momento não foi importante, também justificaram as suas respostas (Tabela 2).

Tabela 2 - Síntese das respostas emitidas pelos alunos na segunda questão

\begin{tabular}{|c|c|c|c|}
\hline & Feedback dos alunos & $\begin{array}{l}\text { Número de } \\
\text { ocorrência }\end{array}$ & Frequência \\
\hline \multirow{7}{*}{ 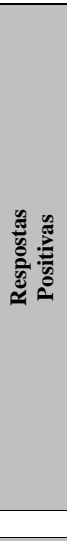 } & $\begin{array}{l}\text { O tempo dedicado à resolução de dúvidas foi } \\
\text { importante, pois foi nele que consegui solucionar } \\
\text { os equívocos cometidos no momento de estudo. }\end{array}$ & 28 & $77,78 \%$ \\
\hline & $\begin{array}{l}\text { O tempo dedicado à resolução de dúvidas foi } \\
\text { importante porque os objetivos do aprendizado } \\
\text { não tinham ficado explícitos quando o professor } \\
\text { disponibilizou o material para o estudo. }\end{array}$ & 2 & $5,56 \%$ \\
\hline & $\begin{array}{l}\text { O tempo dedicado à resolução de dúvidas foi } \\
\text { imprescindível, uma vez que no momento de } \\
\text { estudo (antes da aula) não tive contato com o } \\
\text { professor. }\end{array}$ & 1 & $2,78 \%$ \\
\hline & $\begin{array}{l}\text { O tempo dedicado à resolução de dúvidas foi } \\
\text { importante, pois oportunizou a resoluçãao de }\end{array}$ & & \\
\hline & $\begin{array}{l}\text { dúvidas aos alunos. Além disso, ofereceu uma } \\
\text { visão geral ao professor sobre o nível de } \\
\text { conhecimento dos alunos. }\end{array}$ & 1 & $2,78 \%$ \\
\hline & $\begin{array}{l}\text { O tempo dedicado à resolução de dúvidas foi } \\
\text { importante, porque o material didático não se } \\
\text { aprofundou em algumas questões do conteúdo. }\end{array}$ & 1 & $2,78 \%$ \\
\hline & Total de respostas com aspectos positivos & 33 & $91,67 \%$ \\
\hline \multirow{3}{*}{ 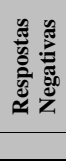 } & $\begin{array}{l}\text { Não, pois os materiais didáticos (vídeos) me } \\
\text { deixaram com muitas dúvidas. }\end{array}$ & 2 & $5,56 \%$ \\
\hline & $\begin{array}{l}\text { Não, porque eu não tinha dúvidas consistentes } \\
\text { pela baixa qualidade dos materiais didáticos } \\
\text { (vídeos) }\end{array}$ & 1 & $2,78 \%$ \\
\hline & Total de respostas com aspectos negativos & 3 & $8,33 \%$ \\
\hline
\end{tabular}

Por fim, na última questão os alunos apresentaram sugestões para melhorar a abordagem. As sugestões são apresentadas na Tabela 3.

Tabela 3 - Sugestões emitadas pelos alunos após o uso da abordagem

\begin{tabular}{lcc}
\hline Feedback dos alunos & $\begin{array}{c}\text { Número de } \\
\text { ocorrência }\end{array}$ & Frequência \\
\hline $\begin{array}{l}\text { Sem sugestões de melhoria. } \\
\begin{array}{l}\text { Poderia melhorar os vídeos que são disponibilizados aos } \\
\text { alunos. }\end{array}\end{array}$ & 8 & $30,56 \%$ \\
$\begin{array}{l}\text { Em sala de aula, o professor poderia dar um exercício } \\
\text { não avaliativo para a turma solucionar, antes de realizar a } \\
\text { atividade avaliativa. }\end{array}$ & 4 & $11,11 \%$ \\
$\begin{array}{l}\text { Seria importante disponibilizar mais materiais didáticos } \\
\text { para o estudo, em diferentes formatos. }\end{array}$ & 4 & $11,11 \%$ \\
$\begin{array}{l}\text { Em sala de aula, o professor poderia apresentar uma } \\
\text { visão geral do conteúdo, não apenas solucionar as } \\
\text { dúvidas dos alunos. }\end{array}$ & 3 & $8,33 \%$ \\
$\begin{array}{l}\text { Seria interessante aumentar o número de exemplos } \\
\text { disponíveis nos vídeos. }\end{array}$ & 3 & $8,33 \%$ \\
$\begin{array}{l}\text { É necessário aumentar o número e diversidade de } \\
\text { exemplos no material didático. Além disso, seria } \\
\text { interessante a realização de exercícios antes da atividade } \\
\text { avaliativa. }\end{array}$ & 1 & $2,78 \%$ \\
$\begin{array}{l}\text { O professor poderia disponibilizar um fórum de } \\
\text { discussão para os alunos solucionarem as dúvidas antes } \\
\text { da aula. Isso ajudaria o aluno que tem dúvida quando } \\
\text { está aprendendo o conteúdo }\end{array}$ & 1 & $2,78 \%$ \\
$\begin{array}{l}\text { O professor poderia ter focado em outro conteúdo como } \\
\text { automatização de teste de software. }\end{array}$ & 1 & $2,78 \%$ \\
\hline
\end{tabular}

A partir dos resultados encontrados, percebe-se que alguns não apreciaram os mecanismos de apoio (vídeos) utilizados no momento "antes da aula". Inclusive, os alunos apresentaram sugestões para melhorar os mecanismos, recomendando a inserção de mais exemplos nos vídeos, a disponibilização de mais materiais e mecanismos com diferentes formatos. Além disso, os alunos sugeriram que o tempo destinado à resolução de dúvidas 
aumentasse, porque muitas dúvidas surgiram durante o aprendizado fora da sala de aula e durante a condução da atividade prática em sala de aula.

As avaliações qualitativas dos alunos auxiliou na percepção que algumas melhorias são necessárias para que Flipped Classroom possa ser aplicado com sucesso para o ensino de teste de software funcional. Após a análise de todas as sugestões, percebeu-se a necessidade de aprimorar a abordagem. Para evoluir a abordagem, as sugestões emitidas pelos estudantes serão consideradas.

\section{Conclusões}

Este artigo apresentou uma abordagem para a definição e uso de Flipped Classroom no ensino de teste de software. A necessidade da abordagem surge uma vez que teste de software é normalmente ensinado com o método tradicional de ensino e este não está conseguindo satisfazer os estudantes atuais. A abordagem foi desenvolvida seguindo os direcionamentos descritos por Bergmann e Sams (2012). Ela é constituída por um planejamento que contém os objetivos de aprendizagem, um conjunto de mecanismos de apoio, um conjunto de atividades e um artefato que representa as ações que são realizadas pelos sujeitos (professor e aluno) envolvidos na implementação da abordagem.

A abordagem foi aplicada em uma disciplina de Engenharia de Software, com o intuito de ser utilizada pelo grupo de alunos. A avaliação foi conduzida no sentido de obter indicadores dos alunos para melhorá-la. A avaliação foi bastante satisfatória, uma vez que foi possível levantar um conjunto de fatores que precisam ser melhorados na abordagem. Os mecanismos de apoio, por exemplo, precisam ser revisados. Os alunos recomendaram que a abordagem precisa ser melhorada, e mecanismos de apoio com diferentes formatos precisam ser disponibilizados aos alunos. É possível que as solicitações dos alunos, por mecanismos com diferentes formatos, possam estar relacionadas aos seus estilos de aprendizagem. Apesar dessa pesquisa ter evitado adotar teorias educacionais, é possível que, se os estilos de aprendizagem fossem identificados antes do professor recomendar os mecanismos, esses problemas poderiam ter sido evitados.

O conjunto de exercícios para complementar a abordagem também precisa ser pensado, uma vez que alguns alunos mencionaram que é importante a resolução de exercícios em sala de aula antes da execução de uma atividade avaliativa, pois é durante a resolução dos exercícios ou condução de uma atividade prática que as principais dúvidas surgem. Outra sugestão é disponibilizar alguns exercícios para os alunos realizarem no momento de estudos. Teste de conhecimentos poderiam ser produzidos e dispobilizados, uma vez que permitira aos alunos um entendimento sobre o conhecimento adquirido.

As observações e indicações dos alunos serão consideradas em um aprimoramento da abordagem. Apesar de um número grande de alunos ter declarado que gostou da abordagem, percebe-se a necessidade de evoluí-la, especialmente por causa das sugestões de melhorias em relação aos mecanismos de apoio (vídeos). Um mecanismo que poderia subsidiar a abordagem, seria um catálogo de recursos educacionais abertos (e.g., vídeos, slides, exercícios) sobre teste de software. Com o catálogo a disposição do professor, ele poderia selecionar com facilidade os materiais didáticos para disponibilizar aos alunos.

\section{Agradecimentos}

Os autores gostariam de agradecer ao CNPq, CAPES e a Fundação de Amparo à Pesquisa do Estado de São Paulo (FAPESP - Processo 2017/10941-8 e Processo 2013/07375-0) pelo apoio financeiro. 


\section{Referências}

BERGMANN, J.; SAMS, A. Flip your classroom: Reach every student in every class every day. Washington, D.C., EUA: International Society for Technology in Education, 2012.

DELAMARO, M.; JINO, M.; MALDONADO, J. Introdução ao Teste de Software. Rio de Janeiro, Brasil: Elsevier, 2016.

FASSBINDER, A. G. O. et al. Tools for the flipped classroom model: An experiment in teacher education. In: 44th Annual Frontiers in Education Conference, 2014. p. $1-8$.

GANNOD, G.; BURGE, J.; HELMICK, M. Using the inverted classroom to teach software engineering. In: 30th International Conference on Software Engineering. 2008. p. 777-786.

GILBOY, M. B.; HEINERICHS, S.; PAZZAGLIA, G. Enhancing student engagement using the flipped classroom. Journal of Nutrition Education and Behavior, v. 47, n. 1, p.109-114, 2015.

HUNG, H.T. Flipping the classroom for english language learners to foster active learning. Computer Assisted Language Learning, v. 28, n. 1, p. 81-96, 2015.

JR, P. L.; ARCURI, A. Recent trends in software testing education: A systematic literature review. In: The Norwegian Conference on Didactics in IT education. 2018.

LEMOS, O. A. L. et al. The impact of software testing education on code reliability: An empirical assessment. Journal of Systems and Software, v. 137, n. 1, p. 497-511, 2018.

MALDONADO, J.; DELAMARO, M.; VINCENZI, A. Automatização de teste de software com ferramentas de software livre. Elsevier Editora Ltda., 2018.

MOZZAQUATRO, P. M. et al. Modelagem de um framework para adaptação de ambientes virtuais de aprendizagem móveis aos diferentes estilos cognitivos. RENOTE -

Revista Novas Tecnologias na Educação, v.7, n.3, p. 1-11, 2009.

MYERS, G. J.; SANDLER, C.; BADGETT, T. The Art of Software Testing. Hoboken, Nova Jersey, EUA: Wiley Publishing, 2011.

PASCHOAL, L. N.; SILVA, L. R.; SOUZA, S. R. S. Abordagem flipped classroom em comparação com o modelo tradicional de ensino: uma investigação empírica no âmbito de teste de software. In: XXVIII Simpósio Brasileiro de Informática na Educação. 2017. p. 476-485.

PASCHOAL, L. N.; SOUZA, S. R. S. A survey on software testing education in Brazil. In: XVII Simpósio Brasileiro de Qualidade de Software. 2018. p. 334-343.

SILVA, V. M.; BARBOSA, E. F.; FASSBINDER, A. G. O. Ferramenta web de apoio à validação de mapas de aprendizagem para MOOCs. In: Workshops do VI Congresso Brasileiro de Informática na Educação. 2017. p. 284-291.

SOSKA, A.; MOTTOK, J.; WOLFF, C. An experimental card game for software testing: Development, design and evaluation of a physical card game to deepen the knowledge of students in academic software testing education. In: 7th IEEE Global Engineering Education Conference.2016. p. 576-584.

TEIXEIRA, G. P. Flipped Classroom: Um contributo para a aprendizagem da líricacamoniana. Dissertação (Mestrado) - Faculdade de Ciências Sociais e Humanas, Universidade Nova de Lisboa, Lisboa, Portugal, 2013. 